\title{
On the Topological Entropy of Nonautonomous Differential Equations
}

\author{
Le Huy Tien, Le Duc Nhien \\ Department of Mathematics, Mechanics and Informatics, Vietnam National University at Hanoi, \\ Hanoi, Vietnam \\ Email: tienlh@viasm.edu.vn,nhien0610@gmail.com
}

How to cite this paper: Tien, L.H. and Nhien, L.D. (2019) On the Topological Entropy of Nonautonomous Differential Equations. Journal of Applied Mathematics and Physics, 7, 418-429.

https://doi.org/10.4236/jamp.2019.72032

Received: January 29, 2019

Accepted: February 23, 2019

Published: February 26, 2019

Copyright (c) 2019 by author(s) and Scientific Research Publishing Inc. This work is licensed under the Creative Commons Attribution International License (CC BY 4.0).

http://creativecommons.org/licenses/by/4.0/

\begin{abstract}
The purpose of this paper is to extend the concept topological entropy to nonautonomous linear systems. Next, we shall give estimation of the topological entropy for the class of bounded linear equations on $\mathbb{R}^{n}$. Finally, we are about to investigate the invariant properties of one through the transformations such as topological conjugacy, topological equivalence and kinematically similar and then show that topological entropy of one is equal to sum of positive Lyapunov characteristic exponents.
\end{abstract}

\section{Keywords}

Kinematically Similar, Topological Conjugacy, Linear Dynamic Equation, Topological Entropy

\section{Introduction and Preliminaries}

Topological entropy, which describes the complexity of a system, plays an important role in topological dynamical systems. It was first induced by Adler et al. [1] as an invariant of topological conjugacy. Later, Bowen [2] gave equivalent definition of topological entropy which measures for how fast the solutions of dynamical system move part. For a linear map on $\mathbb{R}^{n}$, topological entropy is given by the sum of the logarithms of the operator's eigenvalues with absolute value greater than 1, see [2]. Recently, Hoock generates for certain infinite-dimensional linear systems, see [3]. In particular, he also showed that topological entropy of a strongly continuous semigroup is given by sum of real parts of the unstable eigenvalues of the infinitesimal generator, if the unstable part is finite-dimensional. The main result of present paper is a generalization of several results for nonautonomous linear systems in the finite-dimensional case. 
Now we introduce some basic notations for nonautonomous differential equations. Let the linear equation

$$
\dot{x}=A(t) x,
$$

where $A(t)$ is the real matrix function which is uniformly bounded on $\mathbb{R}^{+}$. In this paper, we consider $X(t)$ is a fundamental matrix solution of (1). For the basic theory of this Equation (1), we refer to the book of Dalecki et al. [4]. In order to describe topological entropy for (1), we introduce the concept of spanning and separated sets following [2]. For any $t \in \mathbb{R}^{+}$, define a metrix $d_{t, X}$ on $\mathbb{R}^{n}$ by

$$
d_{t, X}(x, y):=\max _{\tau \in[0, t]}\|X(\tau) x-X(\tau) y\| \text { for all } x, y \in \mathbb{R}^{n} .
$$

Let $K$ be a compact subset of $\mathbb{R}^{n}$. For any $\varepsilon>0$, a subset $R \subset \mathbb{R}^{n}$ is said to be an $(t, \varepsilon)$-spanning set of $K$, if for any $x \in K$ there exists $y \in R$ such that $d_{t, X}(x, y) \leq \varepsilon$. Let $r(t, \varepsilon, K, A, X)$ denote the minimal cardinality of any $(t, \varepsilon)$-spanning set of $K$.

Analogously, a set $S \subset K$ is said to be an $(t, \varepsilon)$-separated set of $K$, if $x, y \in S, \quad x \neq y$, implies $d_{t, X}(x, y)>\varepsilon$. Let $s(t, \varepsilon, K, A, X)$ denote the maximal cardinality of any $(t, \varepsilon)$-separated set of $K$.

Lemma 1.1. Let $X(t)$ is a fundamental matrix solution above. Assume $\varepsilon, t>0$ and $K \subset \mathbb{R}^{n}$ be a compact set. We have that

$$
r(t, \varepsilon, K, A, X) \leq s(t, \varepsilon, K, A, X) \text { and } s(t, 2 \varepsilon, K, A, X) \leq r(t, \varepsilon, K, A, X) .
$$

Proof. Suppose $S$ is the $(t, \varepsilon)$-separated set with maximal cardinality. By definition, if $y \in K$ then $d_{t, X}(x, y) \leq \varepsilon$ for all $x \in S$. Therefore $S$ is the $(t, \varepsilon)$ -spanning, it means the first inequality hold. To prove the second one, we set $R$ is the minimal $(t, \varepsilon)$-spanning set. Then we have

$$
K=\bigcup_{x \in R 0 \leq \tau \leq t} X^{-1}(\tau) B(X(\tau) x, \varepsilon)
$$

where $B(x, r)$ is a ball, centre $x$ and radius $r$. Let $S \subset K$ is the maximal $(t, 2 \varepsilon)$-separated set. If $x_{1}, x_{2} \in \bigcap_{0 \leq \tau \leq t} X^{-1}(\tau) B(X(\tau) y, \varepsilon)$ for some $y \in R$ then

$$
\begin{aligned}
& \left\|X(\tau) x_{1}-X(\tau) x_{2}\right\| \\
& \leq\left\|X(\tau) x_{1}-X(\tau) y\right\|+\left\|X(\tau) y-X(\tau) x_{2}\right\| \leq 2 \varepsilon, \forall \tau \in[0, t] .
\end{aligned}
$$

It means $x_{1}=x_{2}$ (since the definition of $(t, \varepsilon)$-separated set) and hence the second inequality is proved.

By previous lemma, the following definition of topological entropy makes sense.

Definition 1.1. Let $\varepsilon>0$. For a compact set $K \subset \mathbb{R}^{n}$ and $X(t)$ is a fundamental matrix solution of (1), topological entropy for $X(t)$ is given by

$$
\begin{aligned}
h(A, X) & =\sup _{K \text { compact }} \lim _{\varepsilon \rightarrow 0^{+}} \limsup _{\tau \rightarrow \infty} \frac{1}{\tau} \log r(\tau, \varepsilon, K, A, X) \\
& =\sup _{K \text { compact }} \lim _{\varepsilon \rightarrow 0^{+}} \limsup _{\tau \rightarrow \infty} \frac{1}{\tau} \log s(\tau, \varepsilon, K, A, X) .
\end{aligned}
$$


Remark 1.1. If $A(t)=A$ is a constant matrix for all $t \in \mathbb{R}$ then the definition above coincide the definition of A.-M. Hoock (see [3]), i.e. $h(A)=\sum \lambda_{i}$, The sum is taken over all eigenvalues $\lambda_{i}$ of $A$ with $\left|\lambda_{i}\right|>0$.

Remark 1.2. If $Y(t)$ is other fundamental matrix solution of (1) then $h(X)=h(Y)$. Indeed, by [5] there is a converse matrix $C$ such that $X(t)=Y(t) C$. If $X$ is belong to $(t, \varepsilon)$-spanning set of a compact set $K$ for $X(t)$ then $X$ is the same for $Y(t)$. Hence, $h(X) \leq h(Y)$. Similarly, one also have $h(Y) \leq h(X)$. It is our purpose.

If we use A to present the Equation (1), by Remark 1.2, we define the topological entropy for (1), denote $h(A)$, as following

$$
h(A)=h(A, X)
$$

where $X$ is some fundamental matrix solutions.

Remark 1.3. Since all norms on $\mathbb{R}^{n}$ are equivalent so $h(A)$ does not depend on the norm chosen.

We now give an outline of the contents of this paper. In Section 2, one gives the upper estimation for topological entropy for the class of bounded equations. In particular, we are going to show that one is less than $n M$ where $n$ is dimension of space and $M$ upper bounded of $A(t)$ for all $t \in \mathbb{R}^{+}$. In Section 3, we concentrate the invariant property of topological entropy. As consequence, one shall prove that topological entropy of the periodic equations is equal to the sum of all positive Lyapunov characteristic exponents of them. Finally, Section 4, we shall show that topological entropy of (1) is equal to sum of positive Lyapunov characteristic exponents.

\section{Estimation of Topological Entropy for Bounded Linear Equation}

In this section we shall give the estimation of topological entropy for bounded linear equation. We shall begin with the following technique lemma.

Assuming $\mathcal{B}(\delta)=\left\{K \subset \mathbb{R}^{n}: \operatorname{diam}(K)<\delta\right\}$.

Lemma 2.1. Let any $\delta>0$. Then

$$
h(A)=\sup _{K \in \mathcal{B}} h(A, K) .
$$

Proof. It is clear that $\sup _{K \in \mathcal{B}} h(A, K) \leq h(A)$. Converse, we know that for any $K \subset \mathbb{R}^{n}$, one have $K \in \bigcup_{k=1}^{m} K_{i}$ and

$$
s(t, \varepsilon, A, K) \leq \sum_{i=1}^{m} s\left(t, \varepsilon, A, K_{i}\right) .
$$

Fix $t, \varepsilon>0$. Choose $K_{i(t, \varepsilon)}$ such that

$$
s\left(t, \varepsilon, A, K_{i(t, \varepsilon)}\right)=\max _{1 \leq i \leq m} s\left(t, \varepsilon, A, K_{i}\right) .
$$

Then

$$
s(t, \varepsilon, A, K) \leq m \cdot s\left(t, \varepsilon, A, K_{i}\right)
$$


and so

$$
s(t, \varepsilon, A, K) \leq \log m+\log s\left(t, \varepsilon, A, K_{i}\right) .
$$

Since there are infinity sets $K_{i}$, so, we can choose $t_{k} \rightarrow \infty$ such that

$$
\lim _{k \rightarrow \infty} \frac{1}{t_{k}} \log s\left(t_{k}, \varepsilon, A, K\right)=\limsup _{t \rightarrow \infty} \frac{1}{t} \log s(t, \varepsilon, A, K)
$$

and $K_{i\left(t_{k}, \varepsilon\right)}$ is fixed set (i.e. $K_{i\left(t_{k}, \varepsilon\right)}=K_{i(\varepsilon)}$ for any $k$ ). Therefore $s(\varepsilon, A, K) \leq s\left(\varepsilon, A, K_{i(\varepsilon)}\right)$. Similarly, we also can choose $t_{k} \rightarrow 0$ such that $K_{i\left(\varepsilon_{t}\right)}$ is fixed set. Conclusion,

$$
h(A, K) \leq \max _{1 \leq i \leq m} h\left(A, K_{i}\right) .
$$

Finally, any compact subsets $K$ of $\mathbb{R}^{n}$ can be covered by a finite number of balls $B_{1}, \cdots, B_{m}$ of diameter $\frac{\delta}{2}$ and hence

$$
h(A, K) \leq \max _{1 \leq i \leq m} h\left(A, K \cap \overline{B_{i}}\right),
$$

which give the relation (2).

The following theorem is the main theorem in this section.

Theorem 2.1. Assume the Equation (1) has matrix function $A(t)$ satisfies $\|A(t)\| \leq M$ for all $t \in \mathbb{R}^{+}$. Then

$$
h(A) \leq M n,
$$

where $n$ is a dimension of matrix $A(t)$.

Proof. Let $m$ is the Lebesgue measure on $\mathbb{R}^{n}, X(t)$ a fundamental matrix solutions of (1). First of all, we is proving the following claim

$$
h(A)=\lim _{\varepsilon \rightarrow 0} \liminf _{t \rightarrow \infty} \frac{1}{t} \log m\left(\bigcap_{0 \leq \tau \leq t} X^{-1}(\tau) B(0, \varepsilon)\right) .
$$

where we denote $B(a, r)$ is the ball whose centre at a with radius $r$. Indeed, let $K$ is a compact subset of $\mathbb{R}^{n}$ with $m(K)>0$. If $R$ is a $(t, \varepsilon)$-spanning set of $K$ then

$$
K \subset \bigcap_{x \in S x \leq \tau \leq t} X^{-1}(\tau) B(X(\tau) x, \varepsilon)=\bigcap_{x \in S}\left(x+\bigcap_{0 \leq \tau \leq t} X^{-1}(\tau) B(0, \varepsilon)\right) .
$$

It implies

$$
\begin{aligned}
& m(K) \leq r(t, \varepsilon, A, K) \cdot m\left(\bigcap_{0 \leq \tau \leq t} X^{-1}(\tau) B(0, \varepsilon)\right) \\
& \Leftrightarrow \frac{1}{t} \log m(K)-\frac{1}{t} \log m\left(\bigcap_{0 \leq \tau \leq t} X^{-1}(\tau) B(0, \varepsilon)\right) \leq \frac{1}{t} \log r(t, \varepsilon, A, K) \\
& \Leftrightarrow \liminf _{t \rightarrow \infty} \frac{1}{t} \log m\left(\bigcap_{0 \leq \tau \leq t} X^{-1}(\tau) B(0, \varepsilon)\right) \leq \limsup _{t \rightarrow \infty} \frac{1}{t} \log r(t, \varepsilon, A, K) .
\end{aligned}
$$

Hence,

$$
\lim _{\varepsilon \rightarrow 0^{+}} \liminf _{t \rightarrow \infty} \frac{1}{t} \log m\left(\bigcap_{0 \leq \tau \leq t} X^{-1}(\tau) B(0, \varepsilon)\right) \leq \lim _{\varepsilon \rightarrow 0^{+}} \limsup _{t \rightarrow \infty} \frac{1}{t} \log r(t, \varepsilon, A, K) .
$$

The last relation is true for all the compact sets $K$ in $\mathbb{R}^{n}$. It means 


$$
h(A) \geq \lim _{\varepsilon \rightarrow 0^{+}} \liminf _{t \rightarrow \infty} \frac{1}{t} \log m\left(\bigcap_{0 \leq \tau \leq t} X^{-1}(\tau) B(0, \varepsilon)\right) .
$$

To prove the converse inequality, suppose $K \in \mathcal{B} \boxplus \delta \otimes$ with $\delta$ is a arbitrary number such that $\delta<1$. Suppose $S$ is an $(t, \varepsilon)$-separated subset of $K$. Then

$$
\left(\bigcap_{0 \leq \tau \leq t} X^{-1}(\tau) B\left(X(\tau) x, \frac{\varepsilon}{2}\right)\right) \cap\left(\bigcap_{0 \leq \tau \leq t} X^{-1}(\tau) B\left(X(\tau) y, \frac{\varepsilon}{2}\right)\right)=\varnothing .
$$

for all $x \neq y$ in $K$. The well-known result that

$$
V_{n}(r)=\frac{\pi^{\frac{n}{2}}}{\Gamma\left(\frac{n}{2}+1\right)} r^{n},
$$

where $\Gamma$ is Euler's gamma function, is the volume for ball of radius $n$. We have

$$
\begin{aligned}
V(1+\varepsilon) & \geq m\left(\bigcup_{x \in S 0 \leq \tau \leq t} X^{-1}(\tau) B\left(X(\tau) x, \frac{\varepsilon}{2}\right)\right) \\
& =m\left(\bigcup_{x \in S}\left(x+\bigcap_{0 \leq \tau \leq t} X^{-1}(\tau) B\left(0, \frac{\varepsilon}{2}\right)\right)\right) \\
& =s(t, \varepsilon, A, K) \cdot m\left(\bigcap_{0 \leq \tau \leq t} X^{-1}(\tau) B\left(0, \frac{\varepsilon}{2}\right)\right) .
\end{aligned}
$$

Therefore,

$$
\begin{aligned}
& \frac{1}{t} \log s(t, \varepsilon, A, K) \leq \frac{\log V(1+\varepsilon)}{t}-\frac{1}{t} \log m\left(\bigcap_{0 \leq \tau \leq t} X^{-1}(\tau) B\left(0, \frac{\varepsilon}{2}\right)\right) \\
& \Leftrightarrow \lim _{\varepsilon \rightarrow 0^{+}} \limsup _{t \rightarrow \infty} \frac{1}{t} \log s(t, \varepsilon, A, K) \leq \lim _{\varepsilon \rightarrow 0^{+}} \liminf _{t \rightarrow \infty} \frac{1}{t} \log m\left(\bigcap_{0 \leq \tau \leq t} X^{-1}(\tau) B\left(0, \frac{\varepsilon}{2}\right)\right) .
\end{aligned}
$$

Because the last inequality hold for all $K \in \mathcal{B}(\delta)$, by Lemma 2.1, we obtain

$$
h(A) \leq \lim _{\varepsilon \rightarrow 0^{+}} \liminf _{t \rightarrow \infty} \frac{1}{t} m\left(\bigcap_{0 \leq \tau \leq t} X^{-1}(\tau) B(0, \varepsilon)\right) .
$$

From (4) and (6), the desired our claim hold. For any $x \in \mathbb{R}^{n}$, one have

$$
\|X(t) x\| \leq\|X(t)\|\|x\|, \quad\left(\forall t \in \mathbb{R}^{+}\right) .
$$

Therefore,

$$
B\left(0, \frac{\varepsilon}{\|X(t)\|}\right) \subset X^{-1}(t) B(0, \varepsilon), \quad\left(\forall t \in \mathbb{R}^{+}\right) .
$$

Hence,

$$
B\left(0, \frac{\varepsilon}{\|X(t)\|}\right) \subset\left(\bigcap_{0 \leq \tau \leq t} X^{-1}(\tau) B(0, \varepsilon)\right)
$$

It leads to

$$
-\frac{1}{t} \log \|X(t)\|^{n}+\frac{1}{t} \log m(B(0, \varepsilon)) \leq \frac{1}{t} \log m\left(\bigcap_{0 \leq \tau \leq t} X^{-1}(\tau) B(0, \varepsilon)\right) \quad \text { (by (5)) }
$$


or

$$
\liminf _{t \rightarrow \infty} \frac{1}{t} \log m\left(\bigcap_{0 \leq \tau \leq t} X^{-1}(\tau) B(0, \varepsilon)\right) \leq \limsup _{t \rightarrow \infty} \frac{n}{t} \log \|X(t)\|
$$

On the other hand, $\|X(t)\| \leq \mathrm{e}^{M t}$ (see J. L. Dalecki [4]), let $\varepsilon \rightarrow 0$, the last inequality becomes

$$
\lim _{\varepsilon \rightarrow 0} \liminf _{t \rightarrow \infty} \frac{1}{t} \log m\left(\bigcap_{0 \leq \tau \leq t} X^{-1}(\tau) B(0, \varepsilon)\right) \leq M n .
$$

Compare with the claim (3), the desired inequality hold.

\section{Topological Entropy and the Transformations}

Let the equation

$$
\dot{x}=B(t) x
$$

where $B$ is the real matrix function which is also uniformly bounded on $\mathbb{R}^{+}$. Let $X(t), Y(t)$ are fundamental matrix solutions of (1) and (7), respectively. The solutions of the Equations (1) and (7) are said to be topological conjugate if there is a homeomorphism $h: \mathbb{R}^{n} \rightarrow \mathbb{R}^{n}$ such that

$$
h \circ X(t) x=Y(t) \circ h(x),
$$

for every $t \in \mathbb{R}$ and $x \in \mathbb{R}^{n}$.

To start this section, we give the question: Is topological entropy invariant property with the topological conjugacy? The first, one considers the simple example. Let the two equations, namely $A, B$, corresponding,

$$
\dot{x}=\left(\begin{array}{cc}
a & 0 \\
0 & -b
\end{array}\right) x \quad \text { and } \quad \dot{y}=\left(\begin{array}{cc}
c & 0 \\
0 & -d
\end{array}\right) y,
$$

where $a, b, c, d>0$ and $a \neq c$. As in [3], A.-M. Hoock shown that $h(A)=a \neq h(B)=c$. On the other hand, by Theorem 2.50 in [5] two the equations above are topological conjugacy. Hence, topological entropy is not invariant property with the topological conjugacy. The following, we shall give a compare critical of topological entropy in term of homeomorphism $h$ and a sufficient condition of homeomorphism $h$ such that topological entropy is invariant.

Proposition 3.1. Let $X(t), Y(t)$ are fundamental matrix solutions of (1) and (7), respectively. Assuming there exists homeomorphism $h: \mathbb{R}^{n} \rightarrow \mathbb{R}^{n}$ satisfies $h \circ X=Y \circ h$. Then the following statements hold

1) $h$ is a noncontraction (i.e. $\|x-y\| \leq\|h(x)-h(y)\|)$ then $h(A) \leq h(B)$,

2) $h$ is a nonexpanding (i.e. $\|h(x)-h(y)\| \leq\|x-y\|)$ then $h(B) \leq h(A)$.

Proof. Without lost the generation, we suppose that $h$ is nonexpanding map. Let $K_{A}$ is a compact set in $\mathbb{R}^{n}$ and $S$ is a separated set of $K_{A}$ with Equation (1) which is has cardinality is equal to $s\left(t, \varepsilon, K_{A}, A, X\right)$. Let $x, y \in S$, by definition of $S$, we obtain $d_{t, X}(x, y)>\varepsilon$. Since the hypothesis of (1), one have estimate 


$$
\begin{aligned}
& \max _{0 \leq \tau \leq t}\|Y(\tau) h(x)-Y(\tau) h(x)\| \\
& =\max _{0 \leq \tau \leq t}\|h(X(\tau) x)-h(X(\tau) x)\|=d_{t, X}(x, y)>\varepsilon .
\end{aligned}
$$

Therefore, $h(S)$ is $(t, \varepsilon)$-separated set of $h\left(X_{A}\right)$ with Equation (7). Hence

$$
s\left(t, \varepsilon, K_{A}, A, X\right) \leq s\left(t, \varepsilon, h\left(K_{A}\right), B, Y\right)
$$

It implies

$$
\begin{aligned}
& \sup _{K_{A} \text { compact }} \lim _{\varepsilon \rightarrow 0^{+}} \limsup _{t \rightarrow \infty} \frac{1}{\tau} \log s\left(\tau, \varepsilon, K_{A}, A, X\right) \\
& \leq \sup _{K_{A} \text { compact }} \lim _{\varepsilon \rightarrow 0^{+}} \limsup \frac{1}{\tau} \log s\left(\tau, \varepsilon, h\left(K_{A}\right), B, Y\right) \\
& \leq \sup _{K_{B}} \operatorname{limpact}_{\varepsilon \rightarrow 0^{+}} \limsup _{t \rightarrow \infty} \frac{1}{\tau} \log s\left(\tau, \varepsilon, K_{B}, B, Y\right) \text {. }
\end{aligned}
$$

In other word, $h(A) \leq h(B)$. The proof of (2) is similar.

Corollary 3.1. If $h$ is a isometric homeomorphism such that $h \circ X=Y \circ h$ then

$$
h(A)=h(B)
$$

Remark 3.1. For the case of discrete, topological entropy is invariant to topological conjugacy, but it is no longer true for continuous case, even for the coefficient matrix is constant. This implies that topological entropy becomes more complex in continuous case. In other words, topological conjugacy cannot preserve the speed of the lose information for nonautonomous linear equations.

The following, we are going to consider property of topological entropy with topological equivalence.

We say that (1) and (7) are topological equivalence (see [6]) if there exists a continuous function $h: \mathbb{R} \times \mathbb{R}^{n} \rightarrow \mathbb{R}^{n}$ with the following properties

1) $h(t, 0)=0$ and $h(t, x) \rightarrow 0$ as $x \rightarrow 0$ uniformly with respect to $t$,

2) $h_{t}: \mathbb{R}^{n} \rightarrow \mathbb{R}^{n}$, defined by $h_{t}(x)=h(t, x)$ is a homogeneous for each fixed $t$

3) $g: \mathbb{R} \times \mathbb{R}^{n} \rightarrow \mathbb{R}^{n}$, defined by $g(t, x)=h_{t}^{-1}(x)$, is continuous and has property (1) also,

4) If $x(t)$ is a solution of (1) then $h(t, x(t))$ is a solution of (7).

Remark 3.2. Condition (4) implies the equality

$$
h(t, X(t) x)=Y(t) h(0, x) .
$$

Remark 3.3. A straightforward verification shows that topological equivalence is an equivalence relation in the class of nonautonomous equations.

The Equations (1) and (7) are said to be kinematically similar if there exists a continuous differential invertible matrix function $S(t)$ (called a kinematic similarity) such that $S(t)$ and $S^{-1}(t)$ are bounded and such that the transformation $x=S(t) v$ takes the solutions of (1) on to the solutions of (2).

Remark 3.4. If the Equations (1) and (7) are kinematically similar, then they 
are topological equivalence. Indeed, in the definition of topological equivalence it suffices to set $h(t, x)=S(t) x$ where $S(t)$ is the function realizing the kinematically similarity.

The following theorem presents the sufficient condition of topological equivalence which prevents topological entropy.

Proposition 3.2. Let (1) and (7) are topological equivalence with the homeomorphism $h, g$ satisfy

$$
\|h(t, x)-h(t, y)\| \leq \gamma_{2}(t)\|x-y\|^{\alpha_{1}} \text { and }\|g(t, x)-g(t, y)\| \leq \gamma_{2}(t)\|x-y\|^{\alpha_{2}},
$$

where $\gamma_{1}(t), \gamma_{2}(t)$ are scalar bounded function on $\mathbb{R}^{n}$ and $\alpha_{1}, \alpha_{2}$ positive constants. Then $h(A)=h(B)$.

Proof. Let $X(t), Y(t)$ are fundamental matrix solutions of (1) and (7), respectively. Suppose $K_{A} \subset \mathbb{R}^{n}$ be a compact set and $R_{A}$ is a minimal $(t, \varepsilon, A)$ -spanning of $K_{A}$. Then $h\left(0, R_{A}\right)$ is a minimal $(t, \varepsilon, B)$-spanning of $h\left(0, K_{A}\right)$. Indeed, let any $x \in h\left(0, K_{A}\right)$, by definition of spanning set, there exits $y \in R_{A}$ such that $d_{t, X}(y, g(0, x)) \leq \varepsilon$. We have following estimation

$$
\begin{aligned}
d_{t, Y}(x, h(0, y)) & =\max _{0 \leq \tau \leq t}\|Y(\tau) x-Y(\tau) h(0, y)\| \\
& =\max _{0 \leq \tau \leq t}\|h(\tau, X(\tau) g(0, x))-h(\tau, X(\tau)) y\| \\
& \leq \max _{0 \leq \tau \leq t} \gamma_{1}(\tau)\|X(\tau) g(0, x)-X(\tau) y\|^{\alpha_{1}} \\
& \leq M_{1} d_{t, X}(y, g(0, x)) \leq M_{1} \varepsilon^{\alpha_{1}},
\end{aligned}
$$

where $M_{1}=\sup _{\mathbb{R}^{+}} \gamma_{1}(t)$. It implies

$$
r\left(t, \varepsilon, K_{A}, A, X\right) \leq r\left(t, M_{1} \varepsilon^{\alpha_{1}}, h\left(0, K_{A}\right), B, Y\right) .
$$

Hence,

$$
\lim _{\varepsilon \rightarrow 0^{+}} \limsup _{t \rightarrow \infty} r\left(t, \varepsilon, K_{A}, A, X\right) \leq \lim _{\varepsilon \rightarrow 0^{+}} \limsup _{t \rightarrow \infty} r\left(t, M_{1} \varepsilon^{\alpha_{1}}, h\left(0, K_{A}\right), B, Y\right)
$$

or

$$
h(A) \leq \sup _{K_{A} \text { compact } \varepsilon \rightarrow 0^{+}} \lim _{t \rightarrow \infty} \limsup _{t \rightarrow \infty} r\left(t, M_{1} \varepsilon^{\alpha_{1}}, h\left(0, K_{A}\right), B, Y\right) \leq h(B) .
$$

By the similar proof above, we also have $h(B) \leq h(A)$. The proposition is complete.

Remark 3.5. It is clear that if (1) and (7) are kinematically similar then they satisfy all hypothesis of previous proposition with $\gamma_{1}(t)=S(t), \gamma_{2}(t)=S^{-1}(t)$, $\alpha_{1}=\alpha_{2}=1$ (where $S(t)$ is kinematic similarity). Therefore the class of kinematically similar nonautonomous equations is invariant topological entropy.

Corollary 3.2. If $(1)$ is periodic equation then

$$
h(A)=\sum \lambda_{i},
$$

where the sum take all the positive Lyapunov characteristic exponents of that equation.

Proof. By Theorem 2.3.1 in [5] and from previous remark, we obtain 
$h(A)=h(B)$ where $B(t)$ are a constant matrix. On the other hand, by [3], $h(B)=\sum \lambda_{i}$ where the sum takes all the positive eigenvalues of $B$. Using [5] again, we have the complete proof.

\section{Topological Entropy and Lyapunov Exponents}

In this section, we show that topological entropy of the Equation (1) is equal to sum of positive Lyapunov characteristic exponents.

Given a fundamental matrix solution $X$ of (1), consider the quantities

$$
\lambda_{i}=\limsup _{t \rightarrow+\infty} \frac{1}{t} \log \left\|X(t) e_{i}\right\|, \quad i=1, \cdots, n,
$$

where $e_{i}$ denotes the $i$ th standard unit vector. When $\sum_{i=1}^{n} \lambda_{1}$ is minimized with respect to all possible fundamental matrix solutions, then the $\lambda_{i}$ are called the Lyapunov exponents, or Lyapunov characteristic numbers, and the corresponding fundamental matrix solution is called a normal basic.

In this section, we can always work with a normal basis $X(t)$ which has ordered Lyapunov exponents

$$
-\infty<\lambda_{1} \leq \lambda_{2} \leq \cdots \leq \lambda_{n_{0}-1} \leq 0<\lambda_{n_{0}} \leq \cdots \leq \lambda_{n}<\infty .
$$

With these definitions we get the following theorem.

Theorem 4.1.

$$
h(A)=\sum_{\lambda_{i}>0} \lambda_{i}
$$

Proof. Let $K \in \mathcal{B}(\varepsilon)$ fixed. Assume that we can choose a fix point $x_{K} \in K$ such that $K$ is covered by a box

$$
\operatorname{Box}\left(x_{K}\right)=\left\{x_{K}+\sum_{i=1}^{n} a_{i} X(0) e_{i}:-\varepsilon \leq a_{i} \leq \varepsilon, i=1, \cdots, n\right\},
$$

where $e_{i}$ is the $t^{t h}$ unit vectors. Suppose the fundamental matrix solution is arranged in the order $a$ increase of the Lyapunov exponents. For each $t \geq 0, \beta>0$, we consider the finite subset of $\operatorname{Box}\left(x_{K}\right)$ which is given by

$$
\gamma\left(x_{K}, t, \beta\right)=\left\{x_{K}+\sum_{i=n_{0}}^{n} \frac{j_{i} \varepsilon}{e^{\left(\lambda_{i}+\beta\right) t}} X(0) e_{i}: j_{i}=-\left[e^{\left(\lambda_{i}+\beta\right) t}\right], \cdots,\left[e^{\left(\lambda_{i}+\beta\right) t}\right]\right\} .
$$

Claim 1. The subset $\gamma\left(x_{K}, t, \beta\right)$ is an $(t, n \varepsilon)$-spanning set of $K$.

Proof of Claim 1.

For any $x \in K$ then $x$ can be written the form

$$
x=x_{K}+\sum_{i=1}^{n} a_{i} X(0) e_{i}
$$

for some $-\varepsilon<a_{i}<\varepsilon, i=1, \cdots, n$. For every $\beta>0$ small enough, one choose $k_{i} \in\left\{-\left[e^{\left(\lambda_{i}+\beta\right) t}\right], \cdots,\left[e^{\left(\lambda_{i}+\beta\right) t}\right]\right\}$ such that

$$
\left|a_{i}-\frac{k_{i} \varepsilon}{e^{\left(\lambda_{i}+\beta\right) t}}\right| \leq \frac{\varepsilon}{e^{\left(\lambda_{i}+\beta\right) t}}, \quad\left(i=n_{0}, \cdots, n\right)
$$

We now set 


$$
y=x_{K}+\sum_{i=n_{0}}^{n} \frac{k_{i} \varepsilon}{e^{\left(\lambda_{i}+\beta\right) t}} X(0) e_{i} \in \gamma\left(x_{K}, t, \beta\right),
$$

then for any $0 \leq t_{0} \leq t$ we get

$$
\begin{aligned}
X\left(t_{0}\right) x & =X\left(t_{0}\right) x_{K}+\sum_{i=1}^{n} a_{i} X(t) X(0) e_{i} \\
& =X\left(t_{0}\right) y+\sum_{i=1}^{n_{0}-1} a_{i} X\left(t_{0}\right) X(0) e_{i}+\sum_{i=n_{0}}^{n}\left(a_{i}-\frac{k_{i} \varepsilon}{e^{\left(\lambda_{i}+\beta\right) t}}\right) X\left(t_{0}\right) X(0) e_{i} .
\end{aligned}
$$

From the last equation and definition of Lyapunov characteristic exponents, one obtain

$$
\begin{aligned}
& \left\|X\left(t_{0}\right) x-X\left(t_{0}\right) y\right\| \\
& \leq \sum_{i=1}^{n_{0}-1}\left|a_{i}\right|\left\|X\left(t_{0}\right) X(0) e_{i}\right\|+\sum_{i=n_{0}}^{n}\left|a_{i}-\frac{k_{i} \varepsilon}{e^{\left(\lambda_{i}+\beta\right) t}}\right|\left\|X\left(t_{0}\right) X(0) e_{i}\right\| \\
& \leq \sum_{i=1}^{n_{0}-1} \varepsilon e^{\left(\lambda_{i}+\beta\right) t_{0}}+\sum_{i=n_{0}}^{n} \frac{\varepsilon e^{\left(\lambda_{i}+\beta\right) t_{0}}}{e^{\left(\lambda_{i}+\beta\right) t}}
\end{aligned}
$$

Choose $\beta>0$ small enough such that $\lambda_{i}+\beta<0$ for all $i=1, \cdots, n_{0}-1$. The last inequality implies

$$
\left\|X\left(t_{0}\right) x-X\left(t_{0}\right) y\right\| \leq n \varepsilon, \quad t_{0} \in[0, t] .
$$

Hence, the Claim 1 is proved.

It is clear that $(t, n \varepsilon)$-spanning set $\gamma\left(x_{K}, t, \beta\right)$ have

$$
\# \gamma\left(x_{K}, t, \beta\right) \leq \prod_{i=n_{0}}^{n}\left(2\left[e^{\left(\lambda_{i}+\beta\right) t}\right]+1\right) .
$$

Therefore, we have following estimation

$$
\begin{aligned}
& \limsup _{\tau \rightarrow \infty} \frac{1}{\tau} \log r(\tau, n \varepsilon, K, A, X) \\
& \leq \limsup _{\tau \rightarrow \infty} \frac{1}{\tau} \# \gamma\left(x_{K}, \tau, \beta\right) \\
& \leq \limsup _{\tau \rightarrow \infty} \frac{1}{\tau} \sum_{i=n_{0}}^{n} \log \left(2\left[e^{\left(\lambda_{i}+\beta\right) \tau}\right]+1\right) \\
& \leq \limsup _{\tau \rightarrow \infty} \frac{1}{\tau} \sum_{i=n_{0}}^{n} \log 3\left[e^{\left(\lambda_{i}+\beta\right) \tau}\right] \\
& =n \beta+\sum_{i=n_{0}}^{n} \lambda_{i}
\end{aligned}
$$

Since $\beta$ is a arbitrary small positive constant, we have

$$
h(A)=\sup _{K \text { compact }} \lim _{\varepsilon \rightarrow 0^{+}} \limsup _{\tau \rightarrow \infty} \frac{1}{\tau} \log r(\tau, \varepsilon, K, A, X) \leq \sum_{i=n_{0}}^{n} \lambda_{i} .
$$

To order the reverse inequality, let $B(x, \varepsilon)$ is a ball with centre $x$ and radius $\varepsilon$. Denote

$$
\gamma(x, t)=\left\{x+\sum_{i=n_{0}}^{n} \frac{j_{i} \varepsilon}{2 e^{\lambda_{i} t}} X(0) e_{i}: j_{i}=-\left[e^{\lambda_{i} t}\right], \cdots,\left[e^{\left(\lambda_{i}+\beta\right) t}\right]\right\} .
$$


We would prove the following claim.

Claim 2. The subset $\gamma(x, t)$ is an $\left(t, \varepsilon e^{-\lambda_{n}} / 2\right)$-separated set of the $B(x, \varepsilon)$. Proof of Claim 2.

Let two distinct points in $\gamma(x)$, namely

$$
x=x+\sum_{i=n_{0}}^{n} \frac{j_{i} \varepsilon}{2 e^{\lambda_{i} t}} X(0) e_{i} \text { and } y=x+\sum_{i=n_{0}}^{n} \frac{k_{i} \varepsilon}{2 e^{\lambda_{i} t}} X(0) e_{i}
$$

$$
\begin{aligned}
& \text { for some } j_{i}, k_{i} \in\left\{-\left[e^{\lambda_{i} t}\right], \cdots,\left[e^{\lambda_{i} t}\right]\right\} \text {. Let } t_{0}=t-1 \in[0, t] \text { we get } \\
& \left\|X\left(t_{0}\right) x-X\left(t_{0}\right) y\right\| \\
& \quad=\sum_{i=n_{0}}^{n} \frac{\left|j_{i}-k_{i}\right| \varepsilon}{2 e^{\lambda_{i} t}}\left\|X\left(t_{0}\right) X(0) e_{i}\right\| \\
& \\
& \geq \sum_{i=n_{0}}^{n} \frac{\left|j_{i}-k_{i}\right| \varepsilon}{2 e^{\lambda_{i} t}} e^{\lambda_{i}(t-1)} \\
& \geq \varepsilon e^{-\lambda_{i_{0}}} / 2 \quad\left(\text { where }\left|j_{i_{0}}-k_{i_{0}}\right|>0\right) \\
& \geq \varepsilon e^{-\lambda_{n}} / 2
\end{aligned}
$$

Hence, we obtain

$$
\begin{aligned}
& \limsup _{\tau \rightarrow \infty} \frac{1}{\tau} \log s(\tau, n \varepsilon, B(x, \varepsilon), A, X) \\
& \geq \underset{\tau \rightarrow \infty}{\limsup } \frac{1}{\tau} \# \gamma(x, \tau) \\
& \geq \underset{\tau \rightarrow \infty}{\limsup } \frac{1}{\tau} \sum_{i=n_{0}}^{n} \log \left(2\left[e^{\lambda_{i} \tau}\right]+1\right) \\
& \geq \limsup _{\tau \rightarrow \infty} \frac{1}{\tau} \sum_{i=n_{0}}^{n} \log 3\left[e^{\lambda_{i} \tau}\right] \\
& \geq \sum_{i=n_{0}}^{n} \lambda_{i} .
\end{aligned}
$$

Since $h(A)$ is supermum of $h(A, K)$, take all $K \in \mathcal{B}(\varepsilon)$ so

$$
h(A) \geq \sum_{i=n_{0}}^{n} \lambda_{i} .
$$

Combining (8) and (9), we conclude the proof.

\section{Acknowledgements}

The first author was supported in part by the VNU Project of Vietnam National University No. QG101-15.

\section{Open Questions}

How is the topological entropy for the class of unbounded linear equations on $\mathbb{R}^{n}$ ?

\section{Conflicts of Interest}

The authors declare no conflicts of interest regarding the publication of this paper. 


\section{References}

[1] Adler, R., Konheim, A. and McAndrew, J. (1965) Topological Entropy. Transactions of the American Mathematical Society, 114, 309-319. https://doi.org/10.1090/S0002-9947-1965-0175106-9

[2] Bowen, R. (1971) Entropy for Group Endomorphisms and Homogeneous Space. Transactions of the American Mathematical Society, 153, 401-414. https://doi.org/10.1090/S0002-9947-1971-0274707-X

[3] Hoock, A.-M. (2014) Topological and Invariant Entropy for infinite-Dimensional Linear Systems. Journal of Dynamical and Control Systems, 20, 19-31. https://doi.org/10.1007/s10883-013-9203-6

[4] Dalecki, J.L. and Krein, M.G. (1974) Stability of Solutions of Differential Equation in Banach Space. AMS, Providence.

[5] Barreira, L. and Valls, C. (2012) Ordinary Differential Equations. AMS, Providence. https://doi.org/10.1090/gsm/137

[6] Palmer, K.J. (1979) A Characterization of Exponential Dichotomy in Terms of Topological Equivalence. $J M A A, 69,8-16$. 